\title{
Decentralized Planning of Energy Demand for the Management of Robustness and Discomfort
}

\author{
Evangelos Pournaras, Matteo Vasirani, Robert E. Kooij and Karl Aberer
}

\begin{abstract}
The robustness of Smart Grids is challenged by unpredictable power peaks or temporal demand oscillations that can cause black-outs and increase supply costs. Planning of demand can mitigate these effects and increase robustness. However, the impact on consumers in regards to the discomfort they experience as a result of improving robustness is usually neglected. This paper introduces a decentralized agent-based approach that quantifies and manages the trade-off between robustness and discomfort under demand planning. Eight fitness functions are experimentally evaluated using real data from two operational Smart Grids. These fitness functions can provide different quality of service levels for demand-side energy self-management that capture both robustness and discomfort criteria.
\end{abstract}

Index Terms - robustness, discomfort, planning, demand, tree topology, Smart Grid

\section{INTRODUCTION}

The main operational objective of Smart Grids is to match energy supply and demand. The extent to which supply can meet demand or demand can be adjusted to certain supply is an indication of network and system robustness. Demandside energy management plays a crucial role in robustness as micro-generation via distributed renewable energy resources and technologies such as electrical vehicles make matching supply and demand challenging [1], [2]. Yet, in demand-side energy management, robustness by itself cannot capture the dynamics of Smart Grids as its impact on human factor is often neglected or under-emphasized [3], [2], [4], [5].

This paper claims that improving robustness via demandside energy management causes a level of discomfort in consumers. The discomfort cost that consumers undertake in order to experience a more robust Smart Grid is referred to as quality of service under demand-side energy self-management. The goal of this paper is to quantitatively evaluate trade-offs between robustness and discomfort. These trade-offs can be managed by different fitness functions introduced as modular part of a decentralized demand-side energy management system: EPOS, the Energy Plan Overlay Self-stabilization system [6], [7]. The performance of fitness functions is experimentally evaluated with real data from two operational

Evangelos Pournaras is with the Faculty of Electrical Engineering, Mathematics and Computer Science, Department of Computer Science and Engineering, Delft University of Technology, Delft, The Netherlands. E-mail: e.pournaras@tudelft.nl

Matteo Vasirani, and Karl Aberer are with Distributed Information Systems Laboratory, EPFL, Lausanne, Switzerland. E-mail: \{matteo.vasirani,karlaberer\}@epfl.ch

Robert E. Kooij is with the Faculty of Electrical Engineering, Mathematics and Computer Science, Department of Computer Science and Engineering, Delft University of Technology, Delft, The Netherlands and TNO, Delft, The Netherlands. E-mail: r.e.kooij@tudelft.nl

Manuscript received September 30, 2013; revised ? ?, 2013.
Smart Grids. Results show that quality of service, in regards to robustness and discomfort, is manageable.

This paper is outlined as follows: Section II illustrates the main concepts of decentralized demand planning discussed in this paper. Section III illustrates the plan generation process. Section IV outlines the coordination mechanism used for decentralized demand planning. Section V shows how robustness and discomfort are computed. It also illustrates how the data of two Smart Grid projects are used in the experimental evaluation that follows in Section VI. Finally, Section VII concludes this paper and outlines future work.

\section{Robustness vs Discomfort In DEMAND Planning}

Demand planning of a consumption source is defined in this paper as the computation of a time series with the amount of energy intended for consumption by this source in a future period of time $T$. Consumption sources in demand-side energy management can be defined at different aggregation levels. For example, the household device, the wall outlet, the meter of a house or even the feeder of a neighborhood are all different aggregation levels at which demand can be planned. For simplicity, this paper studies demand-planning at the level of household meters, yet, the approach illustrated in this paper can be extended to other aggregation levels as well.

Planning of demand can be applied as a proactive approach for creating a more homogeneous demand curve via (i) loadshifting and/or (ii) load-adjustment. The former action shifts load from high peak times to low peak times without a significant influence in the average load over time [3]. The latter action decreases (or increases) average load via, for example, incentives mechanisms [4]. Both types of action can be applied to improve robustness by preventing disruptions, such as black-out events, or minimize their impact in case they occur. They can be also used for a more efficient utilization of energy resources, e.g., renewables [8], [9].

On the other hand, discomfort refers to the impact that consumers experience on their lifestyle due to a higher robustness via load-shifting and load-adjustment performed by demandside energy management systems. This paper distinguishes two types of discomfort that consumers may experience: (i) shifting discomfort and (ii) adjustment discomfort. Shifting discomfort is related to the inconvenience experienced by load-shifting. For example, if planned demand suggests the availability of warm water for showering at later or earlier time than the intended one, this is an indication of discomfort. Adjustment discomfort is related to the inconvenience experienced by loadadjustment. For example, if planned demand suggests lower 
demand than the intended one for heating during winter, this is an indication of discomfort. However, if the planned demand is higher than the intended one, this is an indication of negative discomfort, assumed to be perceived as comfort. Section V shows how discomfort can be computed in the context of two operational Smart Grid projects.

Demand-side energy management is often performed in a centralized fashion with utilities companies having a significant level of control in demand planning. This approach raises several issues related to scalability and privacy. Costly investments in computing resources are required by utility companies in order to store and process a large amount of streaming data originated by consumers. Moreover, detailed information about household demand can be used to extract information about the lifestyle of consumers resulting in violation of their privacy. This paper studies an alternative decentralized approach for demand-side self-management: software agents represent the demand preferences of consumers, control their demand by generating a set of possible plans $\mathbf{P}_{i}=\left\{\mathbf{p}_{i}^{1}, \ldots, \mathbf{p}_{i}^{l}\right\}, \forall$ agent $i$, and choose the selected plan $\mathbf{s}_{i} \in \mathbf{P}_{i}$ for execution according to criteria defined by a fitness function. Possible plans are actually alternative demand time series for the same future period of time.

Agents can generate two types of possible plans in regards to the discomfort that these plans cause to consumers when executed: (i) equivalent and (ii) non-equivalent possible plans.

Equivalent possible plans are assumed to have an insignificant and equal impact on the discomfort of consumers. For example, possible plans with an equal level of average demand (over the planning time) may be equivalent. In contrast to lighting sources or television, generating equivalent possible plans is feasible for thermostatically controlled appliances whose operation can be planned without significant disturbance in the lifestyle of consumers [6].

However, possible plans can be non-equivalent as they may cause different levels of discomfort, e.g., possible plans with varied level of average demand (over the planning time). This paper focuses on planning of demand based on non-equivalent possible plans. By adopting non-equivalent possible plans, two opposing objectives need to be met: maximizing robustness of Smart Grids while minimizing discomfort that consumers experience. If consumers need to decrease their demand as a response to a power peak that threatens the stability of Smart Grids, discomfort is unavoidably increased.

\section{LOCAL PLAN GENERATION}

Figure 1 summarizes a high-level overview of the demandside energy self-management approach illustrated in this paper. Possible plans can be locally generated by clustering historical demand data. Clustering groups time series demand data sampled every certain time period, e.g., every day, for a total period of time, e.g., a week or a month. Grouping is based on the computation of a proximity metric such as the Euclidean or the Manhattan distance [10]. The number of clusters is usually part of the clustering parameterization and represents the number of possible plans that agents generate.

The total period of time from which historic data are used as input in clustering can be defined by a sliding clustering

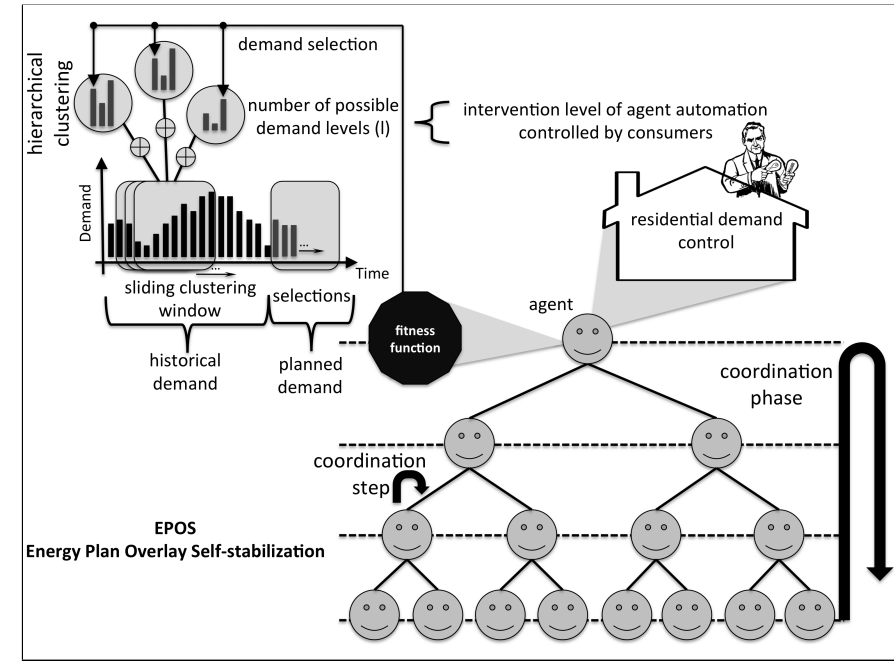

Fig. 1. An overview of the demand-side energy self-management approach illustrated in this paper.

window. For example, the CAISO demand forecasting methodology predicts demand based on the energy consumption of the past 10 days [11]. The same principle can be adopted for the generation of possible plans in a following day.

Each possible plan is devised by computing the representative demand time series of each cluster. More specifically, each possible plan is the medoid of a cluster and is computed by the median of the historical time series that belongs to this cluster. In clustering, the centroid, computed by the mean, is often employed as the center of clusters. However, this paper considers the centroid as not appropriate for demand planning. The centroid is a computed time series that is not necessarily included in the input historical demand data. The historic demand can be used to reason that the centroid is actually a non-possible plan as consumers have not previously devised such a demand configuration via their consumption devices.

A critical aspect in the clustering process is the number of clusters $l$ that corresponds to the number of possible plans. Previous experimental work shows that a higher number of possible plans in demand-side energy management results in improved robustness for Smart Grids [6], [7]. A higher number of possible plans means that agents have a higher degree of freedom to adjust demand according to system objectives. However, a higher number of possible plans increases computational $\operatorname{cost}^{1}$ and causes a lower cluster size on average. A cluster with a lower size results in a devised possible plan that is less representative of past energy consumption. This effect is interpreted as providing a higher level of authority to agents to autonomously reason about the level of household demand and is referred to in this paper as the intervention level of home automation technologies for demand planning. The intervention level $I_{i}^{j}$ of a possible plan $j$ generated by agent $i$ is defined as follows:

\footnotetext{
${ }^{1}$ The increased computational cost concerns the generation process but also the optimization performed by EPOS as illustrated in Section IV.
} 


$$
I_{i}^{j}=1-\frac{C_{i}^{j}}{\sum_{j=1}^{l} C_{i}^{j}}
$$

, where the relative cluster size is computed by the size of the cluster $C_{i}^{j}, j \in[1, l]$ and the sum $\sum_{j=1}^{l} C_{i}^{j}$ of the total number of historic time series sampled for clustering.

\section{Coordinated Plan Selections}

Agents select and execute one of their possible plans to meet different system objectives of Smart Grids. Two types of agent selections are distinguished in this paper: (i) local and (ii) coordinated selections. A local selection of an agent is independent of other agent selections. For example, selecting the plan with the minimum average energy consumption is a local selection that each agent can perform individually. However, for more complex system objectives related to loadshifting, agent selections are interdependent and coordination between agents is required.

Centralized coordination is not a scalable approach as the complexity for computing the optimum combination of agent selections is exponential. More specifically, in a network of $n$ agents with $l$ number of possible plans per agent, the complexity of a brute-force operation is $O\left(l^{n}\right)$. A brute-force operation computes the sum of all combinations between the possible plans of agents. The sum of each combination computed by an agent $i$ is referred to as the combinational plan $\mathbf{c}_{i}^{j}$. This paper focuses on large-scale decentralized coordination of agent selections using EPOS, the Energy Plan Overlay Self-stabilization system [6], [7]. In EPOS, agents are organizationally structured in a tree topology through which they interact and coordinate their selections. EPOS decreases computational complexity to $O\left(l^{c}\right)$, where $c$ is the number of children per agent for a $c$-ary tree. Fault tolerance can be provided with self-organization mechanisms such as AETOS [12] that builds and maintains reconfigurable tree topologies in dynamic distributed environments.

Coordination in EPOS is performed in bottom-up consecutive coordination steps between children and their parents. During a coordination step, the children of a tree level provide to their parents their possible plans together with the summation of all selection performed in the branch underneath. For each agent $i$ with $c$ children, this summation is the aggregate plan $\mathbf{a}_{i}=\sum_{v=1}^{c} \mathbf{a}_{v}=\sum_{h \in\left|\mathbf{B}_{i}\right|} \mathbf{s}_{h}, \forall$ agent $h$ belonging to the branch $\mathbf{B}_{i}$ underneath agent $i$. The possible and aggregate plans are input in a fitness function. The output of the fitness function indicates the selected plan of each child. The process of consecutive coordination steps repeats up to the root that broadcasts to each agent $i$ the global plan $\mathbf{g}=\sum_{i=1}^{n} \mathbf{s}_{i}$ of the system that is the summation of all agent selections. The broadcast completes the coordination phase after which each selected plan $\mathbf{s}_{i}$ can be executed. More details about the algorithm execution and the agent interactions are illustrated in earlier work [6], [7].

This paper studies and evaluates the fitness functions of Table I. Note that the fitness functions based on local selections receive as input local information such as each possible plan $\mathbf{p}_{i}^{j}$ and intervention level $I_{i}^{j}$. MAX-DEMAND and Min-DEMAND are the actual upper and lower bound of demand adjustment. They also represent the maximum adjustment comfort and discomfort that consumers can experience respectively as illustrated in Section V. The fitness functions for coordinated selections receive for input the aggregate plan $\mathbf{a}_{i}$ and each combinational plan $\mathbf{c}_{i}^{j}$. The homogeneity of the planned demand over time is captured by these functions with various metrics such as the standard deviation (Min-Deviations), the relative standard deviation (MIN-RELATIVE-DEviations), the load factor [13] (MAX-LOAD-FACTOR) and the entropy [5] (MAX-EnTRopy).

TABLE I

FITNESS FUNCTIONS FOR DEMAND-SIDE ENERGY MANAGEMENT.

\begin{tabular}{|c|c|c|}
\hline & Fitness Function & Definition \\
\hline \multirow{4}{*}{$\begin{array}{l}\text { Local } \\
\text { Selections }\end{array}$} & RANDOM: & $\operatorname{rand}_{j=1}^{l}(1, \ldots, l)$ \\
\hline & Min-DEMAND: & $\underset{j=1}{\arg \min }\left\{\operatorname{avg}\left(\mathbf{p}_{i}^{j}\right)\right\}$ \\
\hline & MAX-DEMAND: & $\underset{j=1}{\arg \max }\left\{\operatorname{avg}\left(\mathbf{p}_{i}^{j}\right)\right\}$ \\
\hline & MIN-INTERVENTIONS: & $\underset{j=1}{\arg \min }\left(I_{j}\right)$ \\
\hline \multirow{5}{*}{ Coordinated } & Min-DEVIATIONS: & $\underset{j=1}{\arg \min }\left\{\sigma\left(\mathbf{a}_{i}+\mathbf{c}_{i}^{j}\right)\right\}$ \\
\hline & MIN-RELATIVE-DEVIATIONS: & $\underset{j=1}{\arg \min }\left\{\frac{\sigma\left(\mathbf{a}_{i}+\mathbf{c}_{i}^{j}\right)}{\operatorname{avg}\left(\mathbf{a}_{i}+\mathbf{c}_{i}^{j}\right)}\right.$ \\
\hline & \multirow{2}{*}{ MAX-LOAD-FACTOR: } & $l^{c} \int \operatorname{avg}\left(\mathbf{a}_{i}+\mathbf{c}_{i}^{j}\right)$ \\
\hline & & $\underset{j=1}{\arg \max }\left\{\overline{\max \left(\mathbf{a}_{i}+\mathbf{c}_{i}^{j}\right)}\right.$ \\
\hline & MAX-ENTROPY ${ }^{*}:$ & $\underset{j=1}{\arg l^{c}} \max \left(-\sum_{t=1}^{T} u_{i}^{t} \log u_{i}^{t}\right)$ \\
\hline
\end{tabular}

For the purpose of this paper, the functionality of EPOS is significantly extended. More specifically, the contributions of this paper to EPOS are the following: (i) Scope extension from the aggregation level of single devices to the aggregation level of household. (ii) Scope extension from equivalent to nonequivalent possible plans. (iii) Introduction of several other fitness functions besides Min-Deviations and REversingDeviations [6], [7]. (iv) Evaluation of EPOS using real data from operational Smart Grids instead of synthetic data.

\section{VALIDATION IN SMART GRID PROJECTS}

The actual robustness and discomfort that consumers experience via demand planning is validated a posteriori using real consumption data from two operational Smart Grid projects, the Electricity Customer Behavior Trial ${ }^{2}$ in Ireland and the Olympic Peninsula Smart Grid Demonstration ${ }^{3}$ in the USA. The data of the projects are referred to in this paper as CONTROL-DATA $\mathbf{d}$ and are used for (i) generating possible plans from historic CONTROL-DATA and (ii) comparing the future CONTROL-DATA that represent the actual demand with the global plan $\mathrm{g}$.

The possible plans of agents are generated by clustering time series consumption data of the past 10 days that is the length of the sliding clustering window. The number of plans

\footnotetext{
${ }^{2} \mathrm{http}$ ///www.ucd.ie/issda/data/commissionforenergyregulationcer/ (last accessed September 2013)

${ }^{3}$ https://svn.pnl.gov/olypen/
} 
is selected based on two different criteria: (i) By assigning a default number of plans to each agent and (ii) by computing the number of plans based on project data. In the former case, the minimum number of $l=2$ is selected. This number minimizes the intervention level and the computational cost in each agent. In the latter case, the number of possible plans is computed by letting agents reason about the preferences of consumers based on selections they made in the context of each project, e.g., survey answers and temperature setpoints.

A robustness metric is introduced in this paper to compare the demand stabilization achieved with each fitness function. Robustness can be quantified by the distance of each demand value in the computed global plan $\mathbf{g}$ from its average $\operatorname{avg}(\mathbf{g})$ that represents the optimum 'flat' demand curve. This distance can be compared with the respective distance of CONTROLDATA $\mathbf{d}$. The robustness $R$ between the two demand curves is computed by the mean square error as follows:

$$
R= \begin{cases}\frac{1}{T} \sum_{t=1}^{T}\left(\hat{g}^{t}-\hat{d}^{t}\right)^{2} & \text { if } \hat{g}^{t} \geq \hat{d}^{t} \\ \frac{1}{T} \sum_{t=1}^{T}-\left(\hat{g}^{t}-\hat{d}^{t}\right)^{2} & \text { if } \hat{g}^{t}<\hat{d}^{t}\end{cases}
$$

, where:

$$
\hat{g}^{t}=1-\frac{\left|g^{t}-\operatorname{avg}(\mathbf{g})\right|}{\operatorname{avg}(\mathbf{g})} \text { and } \hat{d}^{t}=1-\frac{\left|d^{t}-\operatorname{avg}(\mathbf{d})\right|}{\operatorname{avg}(\mathbf{d})}
$$

Shifting discomfort is computed by the root mean square error between the selected plans and CONTROL-DATA for each agent $i$ :

$$
D_{s}=\sum_{i=1}^{n}\left\{w_{i}^{s} \sqrt{\frac{1}{T} \sum_{t=1}^{T}\left(s_{i}^{t}-d_{i}^{t}\right)^{2}}\right\}
$$

Note that the weight $w_{i}^{s}$ of each agent $i$ quantifies the interest of each consumer for load-shifting. A high value of $w_{i}^{s}$ shows that a consumer is not so self-interested in loadshifting or the impact of shifting discomfort is perceived more 'negative' compared to consumer with a low $w_{i}^{s}$. The value of this weight is selected in the context of the Smart Grid projects illustrated in the rest of this section.

Adjustment discomfort is computed by summing positive and negative errors between the selected plans and CONTROLDATA for each agent $i$ :

$$
D_{a}=\sum_{i=1}^{n} w_{i}^{a} \sum_{t=1}^{T}\left(s_{i}^{t}-d_{i}^{t}\right)
$$

The weight $w_{i}^{a}$ of each agent $i$ is related with how 'negative' different consumers perceive the adjustment discomfort due to demand reduction. Similarly to $w_{i}^{s}$, values are selected within the context of the Smart Grid projects.

\section{A. The Electricity Customer Behavior Trial project}

This project is a cost-benefit analysis that assesses the impact on electricity consumption of consumers in Ireland.
The project ran in the period 2009-2010 with 5000 residential and business consumers participating. The data are cleaned from missing values and filtered out to contain the energy consumption time series of 782 residential consumers that belong to the control group ${ }^{4}$.

Agents reason about the number of possible plans based on the following two questions ${ }^{5}$ :

Question 1. My household may decide to make minor changes to the way we use electricity.

Question 2. My household may decide to make major changes to the way we use electricity.

The answer $a_{q}$ in each of the above question $q$ belongs in the range $[1,5]$, where 1 stands for a strong agreement and 5 stands for a strong disagreement. Algorithm 1 of Appendix A illustrates how agents reason about the number of possible plans they generate ${ }^{6}$. Note that the number of plans computed by this algorithm is referred to in this paper as $l=f_{1}(z=x)$. The seed $z$ is used as a scaling factor in the plan generation process for the different experiments performed.

The weights of robustness and discomfort are computed by the answers of consumers to the following two questions:

Question 3. I am interested in changing the way I use electricity if it helps environment ${ }^{7}$.

Question 4. It is too inconvenient to reduce our usage of electricity $^{8}$.

Based on the range $[1,5]$ of answers, where 1 stands for a strong agreement and 5 stands for a strong disagreement, the weights $w_{i}^{s}$ and $w_{i}^{a}$ for each agent $i$ are computed by normalizing the answers in the range $[0,1]$

\section{B. The Olympic Peninsula Smart Grid Demonstration project}

This project assesses the adjustment of individual energy use based on price signal exchanged within a two-way bidding market [14]. The project concerns the period March 2006 to March 2007 with 112 household participants regionally distributed in the Olympic Peninsula of the USA. The data subset from November 2006 to March 2007 is selected during which the fewest number of missing values is observed. The demand of each consumer is captured every 5 minutes. Demand data are aligned to the sampling rate of the Electricity Customer Behavior Trial project by aggregating 12 consecutive demand bids of each hour to a single hourly demand bid.

\footnotetext{
${ }^{4}$ These consumers are not affected by the dynamic pricing schemes applied for the purpose of the project.

${ }^{5}$ The question block ' 55122 ' of the pre-trial residential survey contains these two questions.

${ }^{6}$ Note that from the total number of 782 residential consumers, 132 of these do not participate in the pre-trial survey. For 116 of these consumers, the question block '54132' of the post-trial survey is used for computing Algorithm 1. This question block is the respective post-trial question block '55122' of the pre-trial survey (My household made minor/major changes to the way we use electricity.). For the final 16 residential consumers that do not participate in neither of the pre-trial nor post-trial surveys, the number of possible plans is computed by the median number of possible plans in the the rest of the 766 consumers.

${ }^{7}$ This is question ' 4331 ' in the residential pre-trial survey.

${ }^{8}$ This is question ' 4352 ' in the residential pre-trial survey.
} 
Demand data are filtered out to contain 29 consumers that (i) either belong to the CONTROL group or have a FIXED type of contract and (ii) have lower than $20 \%$ of their values missing. Two extra consumers are excluded as their demand time series contains a large proportion of zero values. Therefore the final number of consumers used is 27 . The missing values in the final consumers are interpolated by computing the average demand values in the past and future 10 days.

In the context of this project, the demand adjustment is achieved by dynamically modifying the temperature setpoints of various household devices. Motivated by this approach, the number of possible plans $l=f_{2}(z=x)$ is defined by a function that captures the selected temperature setpoints of consumers during project runtime. More specifically, the range of minimum and maximum temperature setpoints selected is normalized to the range $l \in[z, z+4]$ for a given seed $z$.

\section{EXPERIMENTAL EVALUATION}

This section quantitatively evaluates the trade-off between robustness and discomfort under different fitness functions. EPOS is re-engineered as a distributed application of Protopeer [15] that is a prototyping toolkit for large-scale distributed systems. Each coordination phase of EPOS runs for 10 different 3-ary tree topologies. Each topology is built by the AETOS overlay service [12]. AETOS self-organizes agents in different random positions for each tree topology to capture the effect of topological positioning. The effect of different types of tree topologies is evaluated in earlier work [16], [7].

Each coordination phase of EPOS concerns a random day of the week and simulates one demand-response event. An implementation of the hierarchical clustering algorithm [17] in Weka ${ }^{9}$ is used for generating the possible plans of agents.

Two seed values are evaluated for each project ${ }^{10}: z=2, z=$ 3 for the Electricity Customer Behavior Trial project and $z=$ $1, z=2$ for the Olympic Peninsula Smart Grid Demonstration project. The probability distributions for each seed value and weights of discomfort are shown in Appendix A.

\section{A. Robustness vs discomfort}

Table II and III summarize the performance of the fitness functions in each project. Performance is measured by the three metrics introduced in this paper, robustness, shifting discomfort and adjustment discomfort. Three planning generation schemes are evaluated in each project, one static with $l=2$ and two dynamic.

Robustness improves for every fitness function that performs coordinated selections in both projects and every generation scheme. The highest improvement is achieved by the Max-Entropy and Min-Relative-Deviations. MinINTERVENTIONS does not have a significant influence on robustness. Note that as the number of possible plans increases, robustness also increases in average $52 \%$, for $l=f_{1}(z=2)$, $61 \%$ for $l=f_{1}(z=3), 30 \%$, for $l=f_{2}(z=1)$, and $39 \%$,

\footnotetext{
${ }^{9} \mathrm{http}: / /$ www.cs.waikato.ac.nz/ml/weka/ (last accessed September 2013)

${ }^{10}$ Note that if $l \leq 1$, then agents select the median time series from the historic sliding window
}

for $l=f_{2}(z=2)$, confirming earlier findings concerning equivalent possible plans [16], [7].

Shifting discomfort maximally decreases under MINInterventions and Min-Demand. MaX-Demand, MaXENTROPY and RANDOM cause the highest shifting discomfort. The high robustness of MAX-ENTROPY is actually achieved through an increase in shifting discomfort. The lowest shifting discomfort under coordinated selections is achieved by MiNDeviations. Note that shifting discomfort is influenced by the increase in the number of possible plans as follows: $0.4 \%$ average increase for $l=f_{1}(z=2), 0.3 \%$ average decrease for $l=f_{1}(z=3), 14 \%$ average increase for $l=f_{2}(z=1)$ and $11.4 \%$ average increase for $l=f_{2}(z=2)$.

Adjustment discomfort maximally decreases under MAXDemand and MaX-Entropy. Min-Demand and MinDEviations cause the highest adjustment discomfort. Note that the high robustness of Min-Deviations is achieved through an increase in adjustment discomfort, in contrast to MAX-ENTROPY that achieves high robustness by increasing shifting discomfort. The increase in the number of possible plans influences adjustment discomfort as follows: 19\% average increase for $l=f_{1}(z=2), 23 \%$ average increase for $l=f_{1}(z=3), 30.3 \%$ average increase for $l=f_{2}(z=1)$ and $15.9 \%$ average increase for $l=f_{2}(z=2)$.

This section also illustrates the cumulative distribution functions of robustness and discomfort for each fitness function and project. A cumulative distribution function $F_{X}(x)=\operatorname{Pr}(X \leq$ $x$ ) for $X=R, D_{s}$ or $D_{a}$ shows how robustness and discomfort are distributed during the runtime of the projects. Therefore, they provide detailed observations compared to the results of Table II and III. Cumulative distribution functions focus on $f_{1}(z=2)$ and $f_{2}(z=2)$.

Figure 2 illustrates the cumulative distribution functions of robustness for the two projects. The fitness functions that perform coordinated selections are shifted to positive robustness values, whereas, local selections and especially MAX-DEMAND are shifted towards negative robustness values. Note that the Electricity Customer Behavior Trial project concerns data of a higher number of consumers and a longer period of time than the Olympic Peninsula Smart Grid Demonstration project. This explains the higher overlap of the cumulative distribution functions in the second project.

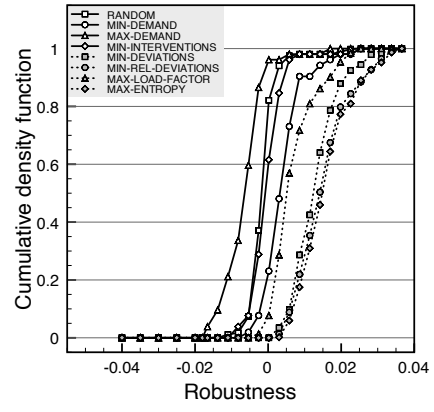

(a) Electricity Customer Behavior Trial project.

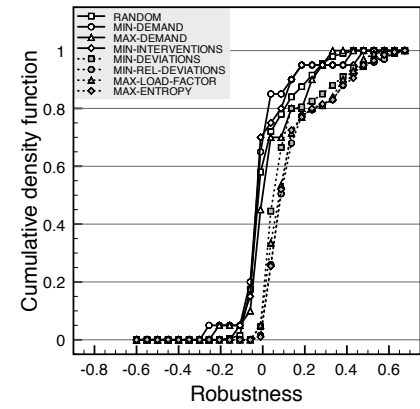

(b) Olympic Peninsula Smart Grid Demonstration project.
Fig. 2. Cumulative distribution functions of robustness $R$ for $z=2$. 
TABLE II

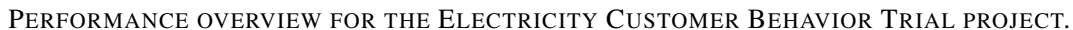

\begin{tabular}{|c|c|c|c|c|c|c|c|c|c|c|}
\hline & Fitness Function & & $R(x * 10$ & & & $D_{s}$ & & & $D_{a}$ & \\
\hline \multirow{5}{*}{$\begin{array}{l}\text { Local } \\
\text { Selections }\end{array}$} & 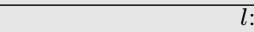 & 2 & $f_{1}(z=2)$ & $f_{1}(z=3)$ & 2 & $f_{1}(z=2)$ & $f_{1}(z=3)$ & 2 & $f_{1}(z=2)$ & $\overline{f_{1}(z=3)}$ \\
\hline & RANDOM: & -0.50 & -0.08 & 0.08 & 83.11 & 81.75 & 82.08 & -349.64 & -327.46 & -311.32 \\
\hline & MIN-DEMAND: & 2.72 & 5.08 & 6.68 & 66.90 & 67.51 & 68.07 & 821.63 & 1053.52 & 1187.48 \\
\hline & MAX-DEMAND: & -5.37 & -5.31 & -5.65 & 99.42 & 96.48 & 97.39 & -1516.07 & -1679.63 & -1809.30 \\
\hline & MIN-INTERVENTIONS: & 0.98 & 1.17 & 1.08 & 65.74 & 66.59 & 67.68 & 594.17 & 609.74 & 586.83 \\
\hline \multirow{4}{*}{$\begin{array}{l}\text { Coordinated } \\
\text { Selections }\end{array}$} & MIN-DEVIATIONS: & 7.79 & 14.28 & 19.18 & 69.03 & 69.51 & 70.18 & 614.72 & 752.65 & 830.76 \\
\hline & MIN-REL-DEVIATIONS: & 9.23 & 16.22 & 21.11 & 74.26 & 74.02 & 74.83 & 143.83 & 169.42 & 196.09 \\
\hline & MAX-LOAD-FACTOR: & 4.83 & 8.17 & 10.68 & 79.30 & 79.30 & 79.30 & -188.86 & -188.86 & -235.40 \\
\hline & MAX-ENTROPY: & 9.64 & 22.05 & 22.04 & 99.42 & 99.42 & 99.42 & -1516.07 & -1516.07 & -1516.07 \\
\hline
\end{tabular}

Low performance $\square$ High performance

TABLE III

Performance overview for the Olympic Peninsula SMARt Grid Demonstration PRoject.

\begin{tabular}{|c|c|c|c|c|c|c|c|c|c|c|}
\hline & Fitness Function & & $R(x * 10$ & & & $D_{s}$ & & & $D_{a}$ & \\
\hline \multirow{5}{*}{$\begin{array}{l}\text { Local } \\
\text { Selections }\end{array}$} & $l:$ & 2 & $f_{2}(z=1)$ & $f_{2}(z=2)$ & 2 & $f_{2}(z=1)$ & $f_{2}(z=2)$ & 2 & $f_{2}(z=1)$ & $f_{2}(z=2)$ \\
\hline & RANDOM: & 21.57 & 47.99 & 47.13 & 47.40 & 40.54 & 42.08 & -108.95 & -80.56 & -96.92 \\
\hline & MIN-DEMAND: & -2.83 & 33.28 & 17.60 & 38.32 & 33.57 & 34.15 & 119.27 & 153.76 & 191.33 \\
\hline & MAX-DEMAND: & 38.37 & 55.34 & 60.51 & 57.03 & 48.18 & 51.37 & -338.55 & -314.20 & -405.29 \\
\hline & MIN-INTERVENTIONS: & -11.36 & 46.76 & 36.68 & 38.59 & 32.19 & 32.60 & 90.26 & 87.86 & 95.12 \\
\hline \multirow{4}{*}{$\begin{array}{l}\text { Coordinated } \\
\text { Selections }\end{array}$} & MIN-DEVIATIONS: & 70.97 & 113.13 & 133.68 & 39.54 & 34.37 & 35.06 & 73.98 & 91.31 & 111.62 \\
\hline & MIN-REL-DEVIATIONS: & 127.38 & 138.38 & 168.16 & 46.0 & 39.48 & 41.09 & -123.49 & -95.60 & -117.12 \\
\hline & MAX-LOAD-FACTOR: & 112.07 & 119.80 & 152.95 & 47.12 & 41.04 & 41.49 & -136.66 & -110.16 & -113.85 \\
\hline & MAX-ENTROPY: & 123.53 & 134.24 & 165.85 & 45.09 & 39.39 & 40.41 & -97.74 & -96.14 & -103.80 \\
\hline
\end{tabular}

Low performance $\square$ High performance

Figure 4 illustrates the cumulative distribution functions of shifting discomfort for the two projects. Min-InTERVENTIONS and Min-DEMAND are positioned to lower shifting discomfort values in contrast to MAX-DEMAND that is clearly positioned to higher values of shifting discomfort. The fitness functions that perform coordinated selections are positioned to higher values compared to Min-INTERVEntions and Min-DEMAND.

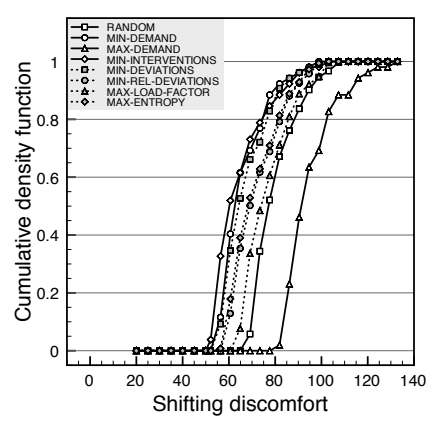

(a) Electricity Customer Behavior Trial project.

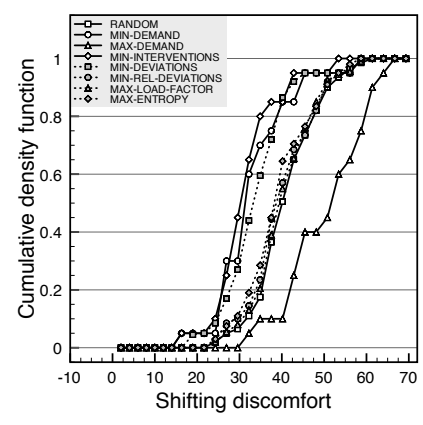

(b) Olympic Peninsula Smart Grid Demonstration project.
Fig. 3. Cumulative distribution functions of shifting $D_{s}$ for $z=2$.

Figure 4 shows that under local selections the cumulative distribution functions of adjustment discomfort are shifted to negative values, yet, Min-Interventions and Min-Demand cause adjustment discomfort and that is why their distributions are shifted to positive values. Under coordinated selections the distributions vary significantly, with MAX-LOAD-FACTOR and MAX-ENTROPY shifted to negative values that cause comfort to consumers, whereas, the rest of the fitness functions are mainly located in between positive and negative values.

This observation can be explained by the fact that coordinated selections acquire a flat demand curve by either

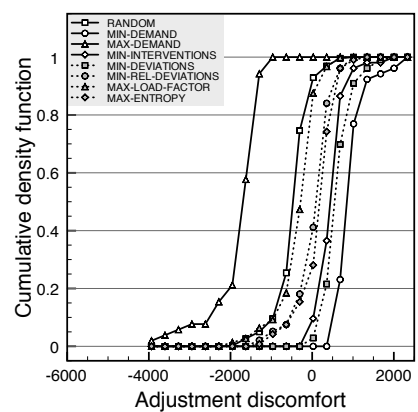

(a) Electricity Customer Behavior Trial project.

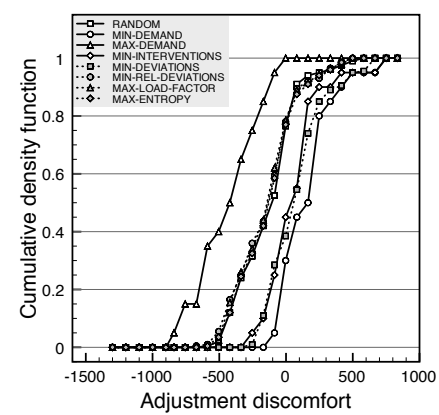

(b) Olympic Peninsula Smart Grid Demonstration project.
Fig. 4. Cumulative distribution functions of adjustment $D_{a}$ for $z=2$.

increasing or decreasing the average demand, e.g., 19/01/2010 and 28/05/2010 respectively for the Electricity Customer Behavior Trial project. Therefore, adjustment discomfort is highly influenced by temporal factors related to the weather and different choices that consumers make in different seasons of a year. The demand curves illustrates in the next section confirm this explanation.

\section{B. Demand curves}

Figure 5 illustrates the demand curves of CONTROL-DATA and the global plans of each fitness function on 19/01/2010 and 28/05/2010 under $l=2$. These data concern the Electricity Customer Behavior Trial project. CONTROL-DATA has two main demand peaks, one low peak in the morning between 06:0008:00 and one high peak in the evening between 17:00-21:00. The morning peak is more distinguishable on 28/05/2010 than $19 / 01 / 2010$, whereas, the evening peak is higher and more distinguishable in the winter day. 

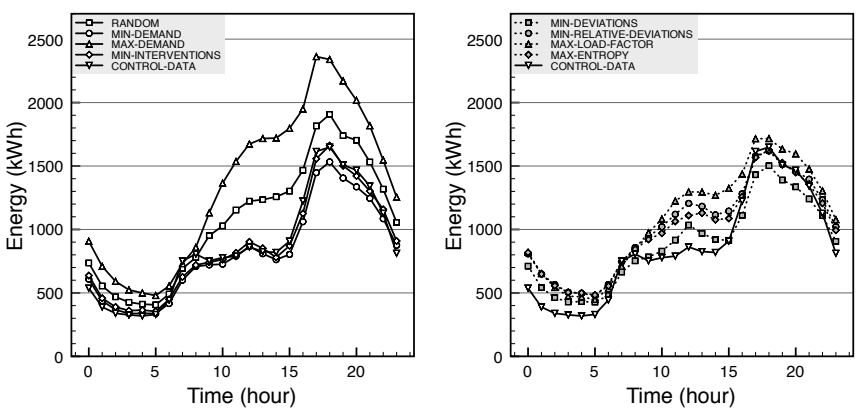

(a) Local selections on $19 / 01 / 2010$.

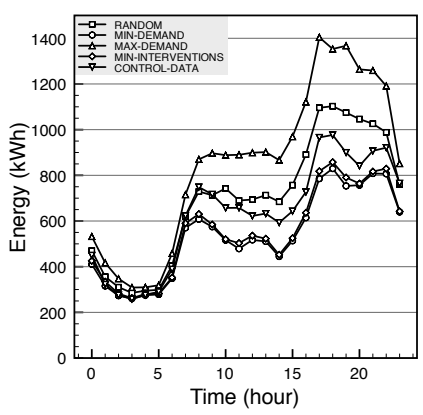

(c) Local $28 / 05 / 2010$ (b) Coordinated selections on $19 / 01 / 2010$

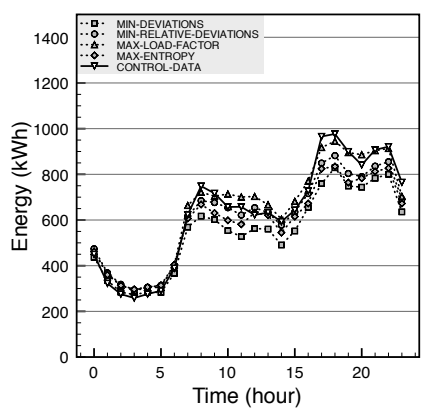

(d) Coordinated selections on 28/05/2010.

Fig. 5. The actual demand curve and the demand of the global plans for the Electricity Customer Behavior Trial project under $l=2$.

Figure 6 illustrates the demand curves of CONTROL-DATA and the global plans of each fitness function on 16/01/2007 for the Olympic Peninsula Smart Grid Demonstration project. The minimum number of possible plans $l=2$ is selected in this case as well. The high winter peak is observed in the morning, with a low evening peak following.

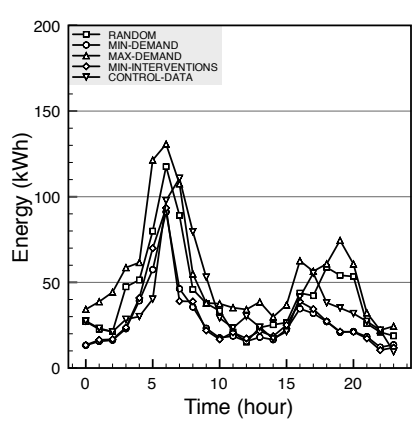

(a) Local selections.

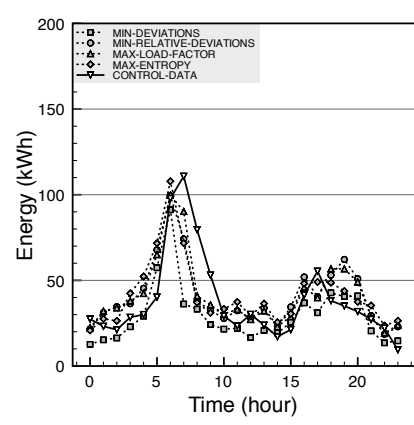

(b) Coordinated selections.

Fig. 6. The actual demand curve and the demand of the global plans for the Olympic Peninsula Smart Grid Demonstration project on 16/01/2007 under $l=2$.

The Max-Demand and Min-Demand in Figure 5a, 5c and $6 \mathrm{a}$ are the upper and lower bounds that form the demand envelope of planning within which the performance of all fitness functions lies. MiN-INTERVENTIONS results in plan selections with low energy consumption. This means that possible plans with extreme high values are not the cluster with the largest size. In Figure 5b and 5d, the global plans are observed

above the CONTROL-DATA during most hours on 19/01/2010 compared to $28 / 05 / 2010$. This means that robustness requires a demand increase for a longer period of time during a winter day compared to a spring day. Note that this demand increase is the actual load-shifting performed to suppress the high power peak. Min-DEVIATIONS decreases the high peak up to $9 \%$ on $19 / 01 / 2010$ and $16 \%$ on $28 / 05 / 2010$ for the Electricity Customer Behavior Trial project and $44 \%$ on 16/01/2007 for the Olympic Peninsula Smart Grid Demonstration project.

\section{Quality of service}

This section shows how performance trade-offs between robustness and discomfort can determine quality of service in demand-side energy self-management. More specifically, assume that consumers, utility companies or system operators need to select one of the fitness functions that satisfies certain robustness and discomfort criteria. Selection is performed as follows:

$$
\underset{o=1}{\arg \max ^{\max }}=\alpha r_{o}^{r}+\beta r_{o}^{s}+\gamma r_{o}^{\mathrm{a}}
$$

, where $r_{o}^{r}, r_{o}^{s}, r_{o}^{a} \in[0,7]$ are the ranks of a fitness function $o \in[1,8]$ for the three respective performance metrics: robustness $R$, shifting discomfort $D_{s}$ and adjustment discomfort $D_{a}$. Ranking is derived by the results of Table II and III with the value of zero corresponding to the lowest performance and the value of 7 to the highest performance. The weights $\alpha, \beta$ and $\gamma$ indicate the relative 'importance' of each performance metric and it holds that $\alpha+\beta+\gamma=1$.

The relation between a certain selection of a fitness function and the threshold values of $\alpha, \beta$ and $\gamma$ that result in this selection can be computed and visualized using decision trees built by the $\mathrm{C} 4.5$ algorithm [18]. Learning is performed by a 10-fold cross-validation of a training set generated using formula (6) with 66 different threshold combinations of $\alpha, \beta$, and $\gamma$ under a step-wise increment of 0.1 .

Figure 7 illustrates the decision tree for the performance results of the Electricity Customer Behavior Trial project. This tree contains two fitness functions that perform local selections (MAX-DEMAND and Min-InTERVEntions) and two fitness functions that perform coordinated selections (MINRelative-Deviations and MaX-Entropy). MAX-DEMAnd is selected when $\beta \leq 0.3$ and $\alpha \leq 0.1$. Min-INTERVEnTIONS is selected under $\beta>0.3$ and $\alpha \leq 0.3$. However for criteria that define high $\alpha$, MaX-Entropy and Min-Relative-Deviations are selected depending on the values of discomfort. In this case, lower shifting discomfort values result in selections of MAX-Entropy over Min-RElative-Deviations.

Figure 8 illustrates the respective decision tree for the Olympic Peninsula Smart Grid Demonstration project. This tree has lower complexity than the tree of the Electricity Customer Behavior Trial project. It provides selections between three fitness functions, MAX-DEMAND, Min-InTERVEntions and Min-Relative-Deviations that are determined by the shifting discomfort and adjustment discomfort.

Note that these decision trees concern the aggregate results of all temporal demand data in each project, yet, these trees 


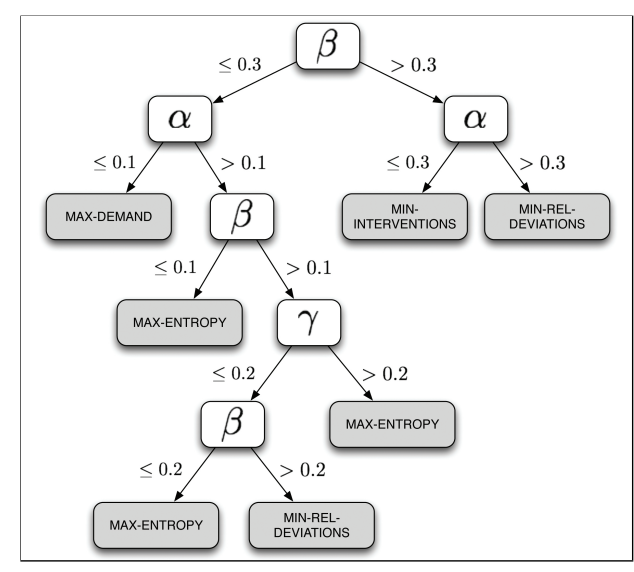

Fig. 7. Decision tree of fitness functions for the Electricity Customer Behavior Trial project.

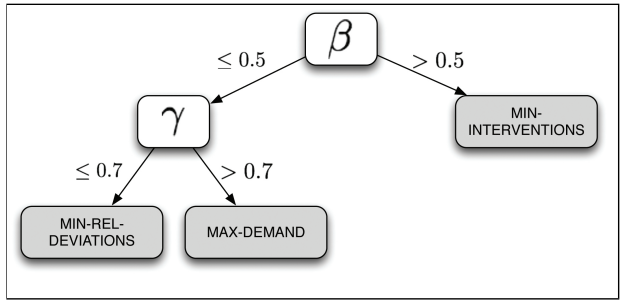

Fig. 8. Decision tree of fitness functions for the Olympic Peninsula Smart Grid Demonstration project.

can be computed for more specific time periods, e.g., seasons or months.

\section{Summary of findings}

The main findings of this paper are summarized as follows:

1) Max-Entropy and Min-Relative-Deviations achieve the highest robustness.

2) Min-Interventions achieves the lowest shifting discomfort and MAX-DEMAND the lowest adjustment discomfort.

3) Min-Deviations achieves the highest peak shavings.

4) A higher number of possible plans increases robustness at a cost of higher discomfort.

5) Peak shaving is achieved either via an overall demand increase or decrease over time.

6) Quality of service under demand-side energy selfmanagement can be managed by decision trees that compute trade-offs between robustness and discomfort.

\section{CONCLUSion AND Future Work}

This paper concludes that the trade-off between robustness and discomfort in demand-side energy self-management is quantifiable, manageable and can provide different quality of service levels. More specifically the experimental validation with real data from two operational Smart Grid project confirms the load-shifting and load-adjustment potential of various fitness functions but also their discomfort impact on consumers. These fitness functions can become a highly modular element of decentralized demand planning mechanisms such as EPOS [6], [7], in future Smart Grids. Other factors related to malicious agents and fairness in discomfort between consumers are part of future work.

\section{ACKNOWLEDGMENT}

This work is funded by the NWO project RobuSmart and the FP7 European project Wattalyst. Authors would like to thank Tri Kurniawan Wijaya for his software support on clustering. Authors are also grateful to the Irish Social Science Data Archive and the Pacific Northwest National Laboratory for providing access to power demand data.

\section{REFERENCES}

[1] P. Palensky and D. Dietrich, "Demand Side Management: Demand Response, Intelligent Energy Systems, and Smart Loads," IEEE Transactions on Industrial Informatics, vol. 7, no. 3, pp. 381-388, Aug. 2011.

[2] G. Strbac, "Demand side management: Benefits and challenges," Energy Policy, vol. 36, no. 12, pp. 4419-4426, Dec. 2008.

[3] M. Stadler, W. Krause, M. Sonnenschein, and U. Vogel, "Modelling and evaluation of control schemes for enhancing load shift of electricity demand for cooling devices," Environmental Modelling \& Software, vol. 24, no. 2, pp. 285-295, Feb. 2009.

[4] P. Joskow and J. Tirole, "Reliability and Competitive Electricity Markets," The RAND Journal of Economics, vol. 38, no. 1, pp. 60-84, May 2007.

[5] H. Aalami, M. P. Moghaddam, and G. Yousefi, "Modeling and prioritizing demand response programs in power markets," Electric Power Systems Research, vol. 80, no. 4, pp. 426 - 435, 2010.

[6] E. Pournaras, M. Warnier, and F. M. T. Brazier, "Local Agent-based Self-stabilisation in Global Resource Utilisation," International Journal of Autonomic Computing, vol. 1, no. 4, pp. 350 - 373, Dec. 2010.

[7] E. Pournaras, "Multi-level reconfigurable self-organization in overlay services," Ph.D. dissertation, Delft University of Technology, March 2013.

[8] M. Erol-Kantarci, B. Kantarci, and H. Mouftah, "Reliable Overlay Topology Design for the Smart Microgrid Network," IEEE Network, vol. 25, no. 5, pp. 38-43, Sep. 2011.

[9] C. Brandstätt, G. Brunekreeft, and K. Jahnke, "How to deal with negative power price spikes? Flexible voluntary curtailment agreements for largescale integration of wind," Energy Policy, vol. 39, no. 6, pp. 3732-3740, Jun. 2011.

[10] R. Giancarlo, G. Lo Bosco, and L. Pinello, "Distance functions, clustering algorithms and microarray data analysis," in Learning and Intelligent Optimization, ser. Lecture Notes in Computer Science, C. Blum and R. Battiti, Eds. Springer Berlin Heidelberg, 2010, vol. 6073, pp. 125138.

[11] A. Sfetsos and C. Siriopoulos, "Time series forecasting of averaged data with efficient use of information," Systems, Man and Cybernetics, Part A: Systems and Humans, IEEE Transactions on, vol. 35, no. 5, pp. 738$745,2005$.

[12] E. Pournaras, M. Warnier, and F. M. T. Brazier, "Adaptation Strategies for Self-management of Tree Overlay Networks," in Proceedings of the 11th IEEE/ACM International Conference on Grid Computing, Grid 2010. Los Alamitos, CA, USA: IEEE, Oct. 2010, pp. 401-409.

[13] R. Chang and C. Lu, "Feeder reconfiguration for load factor improvement," in Power Engineering Society Winter Meeting, 2002. IEEE, vol. 2, 2002, pp. 980-984.

[14] D. J. Hammerstrom, "Part I. Olympic Peninsula Project," Pacific Northwest National Laboratory, Richland, WA, USA, Tech. Rep., Oct. 2007.

[15] W. Galuba, K. Aberer, Z. Despotovic, and W. Kellerer, "ProtoPeer: A P2P Toolkit Bridging the Gap Between Simulation and Live Deployement," in Proceedings of the Second International Conference on Simulation Tools and Techniques, ICST 2009. Gent, Belgium: ACM, Mar. 2009, pp. 1-9.

[16] E. Pournaras, M. Warnier, and F. M. T. Brazier, "Self-Optimised Tree Overlays Using Proximity-Driven Self-Organised Agents," in Complex Intelligent Systems and their Applications, ser. Springer Optimization and its Applications. New York, NY: Springer New York, Aug. 2010, vol. 41 , ch. 7 , pp. 137-161.

[17] A. K. Jain, "Data clustering: 50 years beyond k-means," Pattern Recognition Letters, vol. 31, no. 8, pp. 651 - 666, 2010.

[18] J. Quinlan, C4.5: Programs for Machine Learning, ser. C4.5 - programs for machine learning / J. Ross Quinlan. Morgan Kaufmann Publishers, 1993. 


\section{APPENDIX A}

\section{SUPPLEMENTAL MATERIAL}

Figure 1 illustrates how the number of possible plans is generated for the Electricity Customer Behavior Trial project. The main intuition behind this algorithm is that as the number of plans increases, the intervention level also increases in average. If a consumer chooses in the Question 2 for major changes in his/her electricity, then a higher intervention level is introduced by increasing the number of possible plans (lines 1-6 in Algorithm 1).

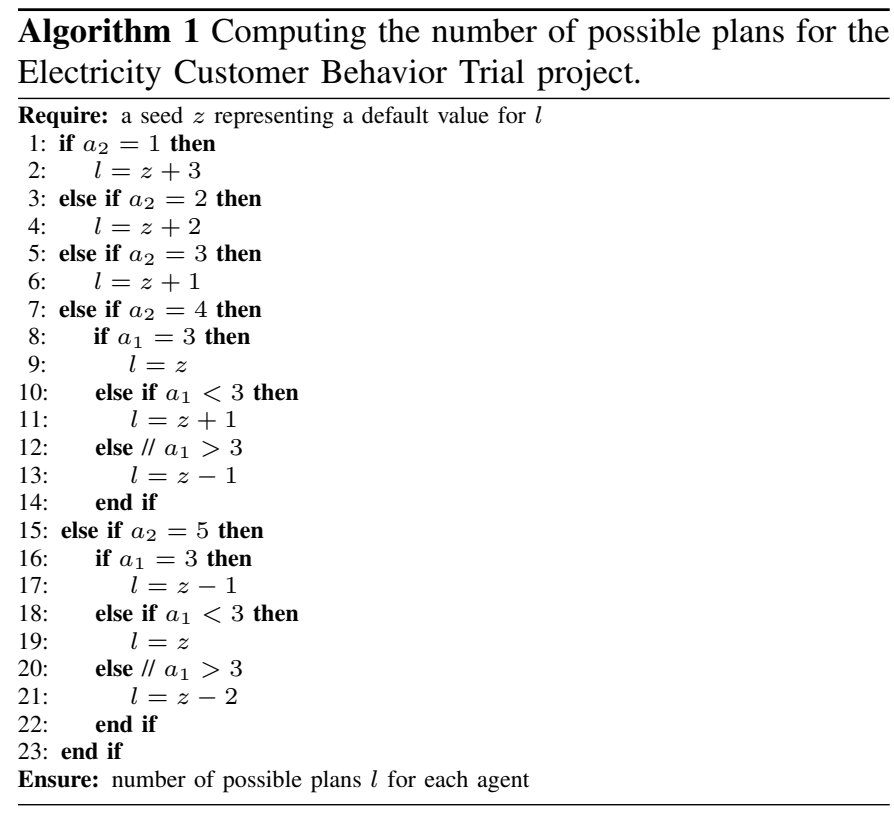

Figure 9 illustrates the probability distribution of the number of possible plans $l$ in the two Smart Grid projects. Two distributions generated by different seed values are shown for each project.

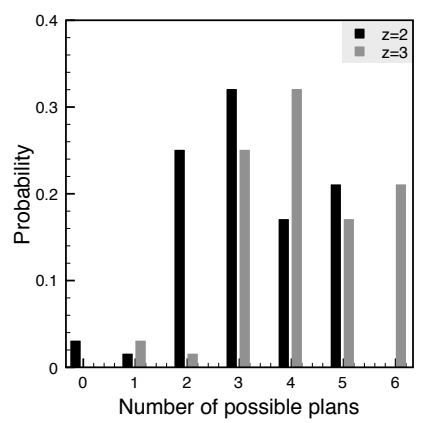

(a) Electricity Customer Behavior Trial project.

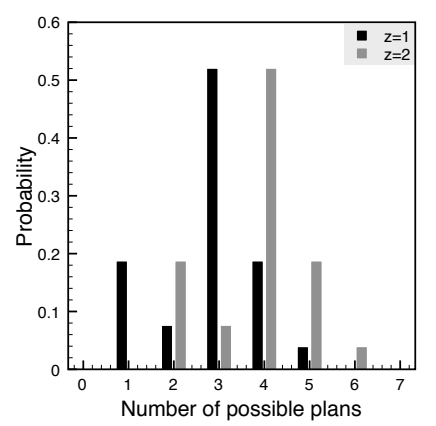

(b) Olympic Peninsula Smart Grid Demonstration project.

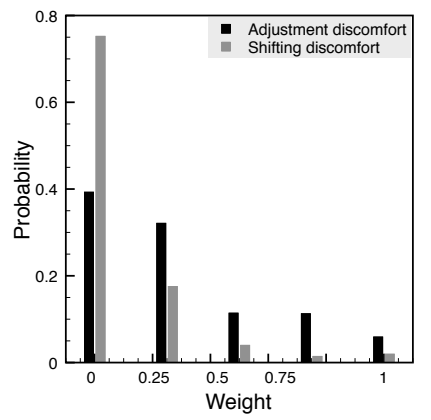

Fig. 10. The probability distribution of shifting $w_{i}^{s}$ and adjustment $w_{i}^{a}$ discomfort weights derived from the pre-trial survey of the Electricity Customer Behavior Trial project.

Fig. 9. The probability distribution for the number of possible plans $l$.

Figure 10 illustrates the probability distribution for the two weights of discomfort based on the answers of consumers in Question 3 and 4. 